\title{
Hybrid synthetic jet actuator with a novel fluidic diode
}

\author{
J. Kordík \& Z. Trávníček \\ Institute of Thermomechanics AS CR, Prague, Czech Republic
}

\begin{abstract}
The paper deals with a new design of the hybrid synthetic jet actuator (HSJ), which is based on a novel fluidic diode. The fluidic diode was tested by a static experiment and its diodicity was evaluated. The same experiment was performed for a second fluidic diode whose planar geometry was taken from the literature and the resultant diodicities were compared. Higher diodicities were achieved with the new design of the diode.

The velocity resonance curves were measured at the nozzle output for two variants of the HSJ with a short or with a long nozzle. After that the volumetric efficiencies of the HSJ were evaluated as functions of the frequency. The highest efficiency (over 80\%) was achieved at the second resonant frequency of the actuator with the long nozzle.

Keywords: synthetic jet, hybrid synthetic jet, rectification effect.
\end{abstract}

\section{Introduction}

Hybrid synthetic jets, which were first introduced in [1-4], combine the usual synthetic jet and valveless pump principles. The valveless pump [5-10] is a pumping device consisting of a chamber equipped with a moving piston or diaphragm, the chamber is connected to a fluidic diode and a nozzle. The orientation of the fluidic diode is adjusted so that its forward direction is oriented toward the inside of the chamber. Once the diaphragm oscillates, a nonzero net mass flow is created in the nozzle (rectification effect). Unlike the valveless pump, the nozzle of the hybrid synthetic jet actuator (HSJA) issues into ambience where the hybrid synthetic jet (HSJ) is formed.

The experimental investigation offers many unexplored possibilities, as hybrid synthetic jets represent a new research topic, with only a few papers dedicated 
to their study $[1-4,11-15]$. The motivation of the present work is based on improvements in the rectification effect of a hybrid synthetic actuator, which is desirable particularly for the augmentation of convective heat transfer of the jet. To quantify this flow rectification, the volumetric efficiency of the actuator is defined.

\section{Hybrid synthetic jet principles}

Figure 1 shows two basic working modes of the valveless pump on which the hybrid synthetic jet actuators (HSJAs) are based. In the first mode, the supply mode, the piston (or diaphragm) of the valveless pump moves left, and a fluid is sucked in. A conical duct element, which was chosen as the fluidic diode here, has a low hydraulic resistance during the inward flow (forward direction), and at the end of the supply cycle, a fluid volume $V_{\mathrm{SD}}$ is supplied into the chamber through it. The fluid volume sucked into the chamber through the nozzle is denoted $V_{\mathrm{SN}}$.

In the pump mode, the fluid is extruded through the fluidic diode, which possesses a higher hydraulic resistance during the outward flow (reverse direction). This causes the fluid volume $V_{\mathrm{PD}}$ passed during the pump cycle through the diode to be smaller than the $V_{\mathrm{SD}}$, and the volume pumped through the nozzle $V_{\mathrm{PN}}$ is larger than the sucked volume in the supply mode $V_{\mathrm{SN}}$. Periodic cycling of the pump and supply modes results in a nonzero net flow in both elements, the diode and nozzle. A measure of the pumping efficiency is the ratio defined in [5] (volumetric efficiency):

$$
\varepsilon_{\mathrm{V}}=\frac{V_{\mathrm{PN}}-V_{\mathrm{SN}}}{V_{\mathrm{SD}}+V_{\mathrm{SN}}} .
$$

Compared with the valveless pump, the nozzle of the HSJA is terminated, and the flow issues into ambience creating flow structure similar to the synthetic jet. The flow structure is called a hybrid synthetic jet (HSJ) and was studied, for example, in $[3,4]$. The paper [4] also introduces another definition of the volumetric efficiency

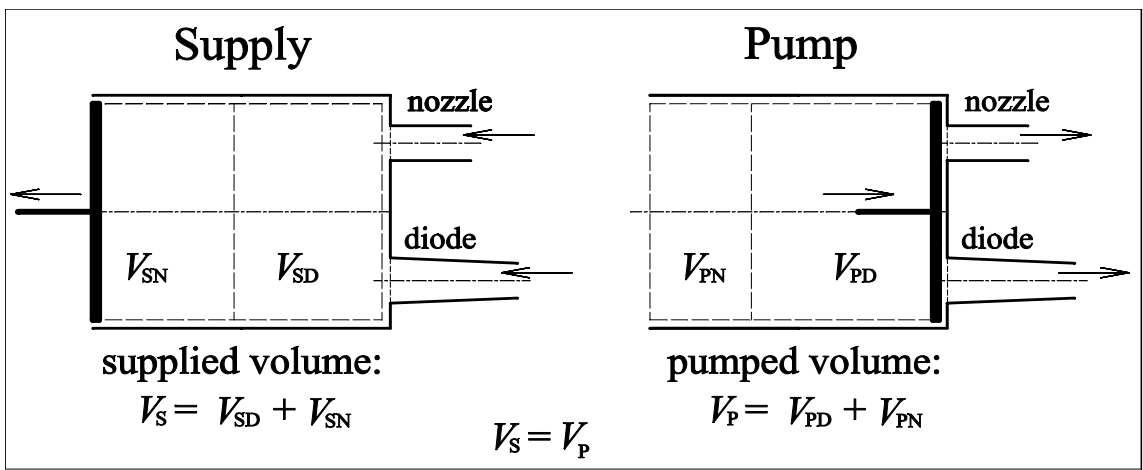

Figure 1: A sketch of the valveless pump (or the HSJA). 
for the hybrid synthetic jet:

$$
\varepsilon_{\mathrm{N}}=\frac{V_{\mathrm{PN}}-V_{\mathrm{SN}}}{V_{\mathrm{PN}}+V_{\mathrm{SN}}} .
$$

This is a more user-friendly definition because the $\varepsilon_{\mathrm{N}}$ is based on HSJ measurement only, without (extremely complicated) measurement of a fluid flux through the diode. This definition was also used as a criterion for comparing hybrid synthetic jet actuators (HSJAs) in [14-16]. The efficiency $\varepsilon_{\mathrm{N}}$ is also used for the investigated HSJA in this work. A criterion for the fluidic diode efficiency can be expressed as the ratio of the pressure loss coefficient in the reverse direction $\xi_{o}$ and the pressure loss coefficient in the forward direction $\xi_{i}$. This is called the diodicity (denotation: $D i$ ) [17-20]:

$$
D i=\frac{\xi_{o}}{\xi_{i}} .
$$

\section{Experimental setup and data processing}

\subsection{The design of the hybrid synthetic jet actuator}

The actuator based on the loudspeaker TVM ARN-165-01/4 is comprised of two fluidic diodes D1 and D2 (see Fig. 2) and is projected such that the diodicity of the both fluidic diodes can be tested by static measurement (see the section 4.1). Concurrently, after an easy adaptation of the HSJA, the hybrid synthetic jet can be investigated at the nozzle output. Two nozzles N1 and N2, which differ by length (30 $\mathrm{mm}$ and $150 \mathrm{~mm}$ respectively), are prepared in the actuator. The hybrid synthetic jet based on the fluidic diode D1 is studied separately for each nozzle, this means that the remaining nozzle will be always sealed during the experiments. The fluidic diode D1 was selected by a static experiment as the one with the higher diodicity (see the section 4.1, Fig. 3). The new design for the diode D1 was developed, and the planar geometry of diode D2 was taken from the paper [20]. According to this paper, the diode is expected to have a high diodicity (around $D i \approx 1.6)$. The experimental conditions, selected dimensions, and properties of the HSJA are summarized in Table 1.

\subsection{Data acquisition and hardware setup}

The experiments aimed at evaluating the volumetric efficiencies $\varepsilon_{N}$ of HSJAs were performed using velocity measurement in the actuator nozzle. The velocities were measured by the hot-wire probe 55P16 placed $1 \mathrm{~mm}$ inward from the nozzle exiting area along the nozzle axis. The hot-wire signal from the anemometer MiniCTA 54T30 DANTEC was sampled in the data acquisition device NI-PCI 6023E. The sampling frequency was set to $10 \mathrm{kHz}$, and the number of samples was 65536 . The hot-wire probe was calibrated in the velocity range $0.2-50 \mathrm{~m} / \mathrm{s}$ after each two hours of continuous operation. The maximal relative uncertainty of single velocity sample was $17 \%$, usual value of the relative uncertainty was under $5 \%$ 


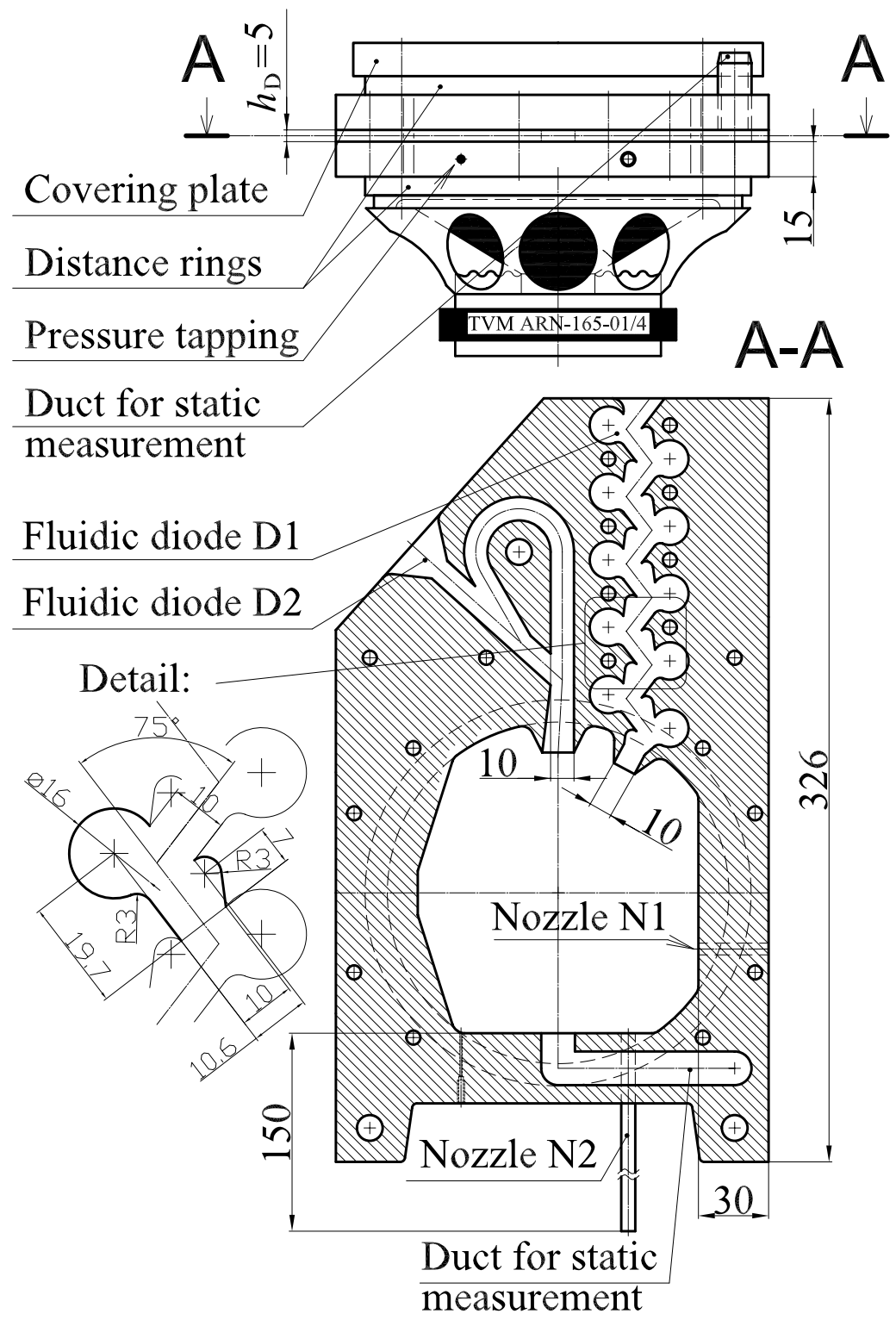

Figure 2: The present hybrid synthetic jet actuator.

for velocities in range 2-50 m/s. A more detailed description of the anemometer settings, probe calibration method, and uncertainty evaluation can be found in [21].

During the velocity measurement the loudspeaker of the actuator was supplied by a harmonic electrical current whose effective value $0.8 \mathrm{~A}$ was kept constant and the frequency of the current was a variable parameter. 
Table 1: The parameters of the experiment.

\begin{tabular}{|c|c|c|}
\hline Dimensions & Dimensions: & Value/formula \\
\hline$D_{0}=2 R_{0}$ & $(\mathrm{~mm})$ & 131 (diaphragm diameter) \\
\hline$D_{2}=2 R_{2}$ & $(\mathrm{~mm})$ & 5 (nozzle diameter) \\
\hline$h_{\mathrm{D}}$ & $(\mathrm{mm})$ & 5 (diode height) \\
\hline$w_{\mathrm{D}}$ & $(\mathrm{mm})$ & 10 (diode width) \\
\hline$L_{2}$ & $(\mathrm{~mm})$ & 30 or 150 (lengths of nozzles N1 or N2) \\
\hline \multicolumn{3}{|c|}{ Fluid properties: } \\
\hline$r$ & $(\mathrm{~J} /(\mathrm{kg} \cdot \mathrm{K}))$ & 287 (specific gas constant) \\
\hline$\kappa$ & (1) & 1.4 (specific heat ratio) \\
\hline$p_{\mathrm{b}}$ & $(\mathrm{Pa})$ & 98900 (barometric pressure) \\
\hline$T$ & $(\mathrm{~K})$ & 292.15 (air temperature) \\
\hline$\rho$ & $\left(\mathrm{kg} / \mathrm{m}^{3}\right)$ & $\frac{p_{\mathrm{b}}}{r T}=1.18$ (air density) \\
\hline & $\left(\mathrm{m}^{2} / \mathrm{s}\right)$ & $1.55 \cdot 10^{-5}$ (kinematic viscosity) \\
\hline \multicolumn{3}{|c|}{ Electric supply: } \\
\hline$I_{\text {eff }}$ & (A) & 0.8 (effective value of current supply) \\
\hline$P$ (power) & $(\mathrm{W})$ & $\approx 2.7$ at $f=20 \mathrm{~Hz}$ (effective value of power supply) \\
\hline
\end{tabular}

\subsection{Hot-wire data reduction}

The volumetric efficiencies $\varepsilon_{\mathrm{N}}$ of the HSJAs were evaluated from the phaseaveraged velocity waveforms with help of additional SJ measurements. The SJ was achieved by sealing off the fluidic diodes ducts of the HSJA, and its velocity waveforms were measured at the same input current, location, and frequencies as the HSJ. The following corrective factor $g_{\mathrm{SJ}}$ was evaluated from the SJ phaseaveraged velocities $U(t)$ :

$$
g_{\mathrm{SJ}}=\frac{\int_{0}^{T_{\mathrm{E}}} U(t) \mathrm{d} t}{\int_{T_{\mathrm{E}}}^{T}|U(t)| \mathrm{d} t} \Leftrightarrow \int_{0}^{T_{\mathrm{E}}} U(t) \mathrm{d} t-g_{\mathrm{SJ}} \int_{T_{\mathrm{E}}}^{T}|U(t)| \mathrm{d} t=0,
$$

where $T_{\mathrm{E}}$ is the extrusion stroke duration time and $T$ is the period. The correction factor $g_{\mathrm{SJ}}$ forces the fulfillment of the continuity equation, which is applied to the centerline velocity measured in the nozzle output. The factor evaluation method is based on the behavior of the SJ/HSJ, which creates slug velocity profiles during the extrusion stroke, and the velocity measured by the hot-wire technique at the nozzle output is in accordance with the cross-sectional averaged velocity. Thus, we obtain a good result for the extruded volume by the integration of the velocity $U(t)$ from 0 to $T_{\mathrm{E}}$, and no correction is necessary.

During the suction stroke, the flow is sucked from a large area behind the nozzle, and the streamlines converge into the nozzle. This outcome causes the 
velocities measured at the nozzle output to not correspond to the cross-sectional averaged velocity and to meet the continuity equation, they must be corrected. The correction factor $g_{\mathrm{SJ}}$ modifies the volume $S_{2} \int_{T_{\mathrm{E}}}^{T}|U(t)| \mathrm{d} t$ so that it is equal to the extruded volume, which is known quite precisely from the integration of $S_{2} U(t)$ from 0 to $T_{\mathrm{E}}\left(S_{2}\right.$ is the nozzle cross-sectional area).

The factor $g_{\mathrm{SJ}}$ obtained from the SJ data is then also applied to the HSJ phaseaveraged velocity, which are acquired under the same conditions and from which the volumetric efficiency is evaluated:

$$
\varepsilon_{\mathrm{N}}=\frac{\int_{0}^{T_{\mathrm{E}}} U(t) \mathrm{d} t-g_{\mathrm{SJ}} \int_{T_{\mathrm{E}}}^{T}|U(t)| \mathrm{d} t}{\int_{0}^{T_{\mathrm{E}}} U(t) \mathrm{d} t+g_{\mathrm{SJ}} \int_{T_{\mathrm{E}}}^{T}|U(t)| \mathrm{d} t} .
$$

\section{Experimental results}

\subsection{Experimental evaluation of diodicities}

The static experiments for the fluidic diodes were performed within the HSJA from Fig. 2. The loss coefficients were measured in the forward and reverse directions for the diodes D1 and D2 at 13 different mass flows in the range $3-301 \mathrm{n} / \mathrm{min}$. The mass flows were measured by a precise digital thermal mass flow meter Bronkhorst EL-FLOW F-201A-50k-AAD-33-V equipped with a particle filter M-422-19-00V. To evaluate a volume flow from the measured mass flow, the flow temperature and barometric pressure were measured.

In order to evaluate loss coefficients, pressure drop along a diode was measured by the digital manometer Greisinger GMH 3156. Pressure taps were located at the ends of the diode in places where the flow velocity was negligible compared with the flow velocity inside the diode. To measure the pressure drops in forward directions, additional flanges with pressure taps were mounted at the exits of the diodes. The flanges were equipped with hose connections for the volume flow supply and comprise a suitable enlarged cross-sectional area for the static pressure measurement.

During the measurement of the pressure drop in the reverse flow direction, the volume flow was supplied by a duct particularly designed for this purpose in the HSJA (see Fig. 2 duct for static measurement). Another arrangement of the HSJA was used for this experiment; the loudspeaker was removed and substituted by a solid plate with a rubber gasket, and the nozzles and remaining fluidic diode were hermetically sealed. The loss coefficients were evaluated using the measured pressure and volume flow $Q$ as:

$$
\begin{aligned}
& \xi_{i}=\frac{2 \Delta p_{\mathrm{d} i} S_{3}^{2}}{\rho Q^{2}}, \\
& \xi_{o}=\frac{2 \Delta p_{\mathrm{do}} S_{3}^{2}}{\rho Q^{2}},
\end{aligned}
$$

where $\Delta p_{\mathrm{d} i}, \Delta p_{\mathrm{d} o}$ are pressure drops in the forward and reverse directions, respectively and $S_{3}$ is the diode cross-sectional area. The loss coefficients 


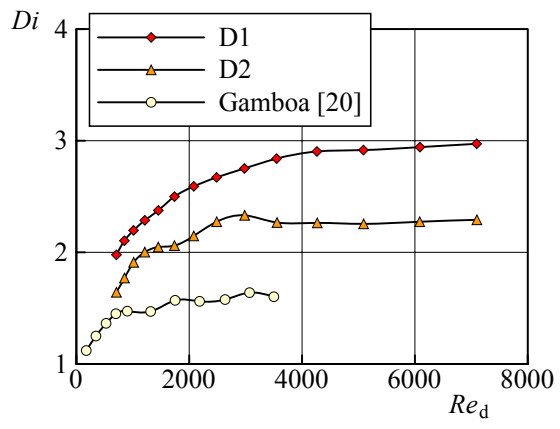

Figure 3: The measured diodicities.

evaluated from the experiments using Eq. (6) were not constant with varying Reynolds number $R e_{\mathrm{d}}$. The Reynolds number was computed as $R e_{\mathrm{d}}=\frac{Q}{h_{\mathrm{D}} \nu}$ (where $h_{\mathrm{D}}$ is the diode duct height, this dimension was the same for both diodes $h_{\mathrm{D}}=5 \mathrm{~mm}-$ see Fig. 2).

The diodicities were evaluated as functions of the Reynolds number $R e_{\mathrm{d}}$ using Eq. (3) and are shown in Fig. 3. All of the measured diodicities increased with the Reynolds number, and the highest values of the diodicity were achieved with the diode D1. For this reason, the fluidic diode D1 is used in unsteady hybrid synthetic jets experiments.

The maximal diodicity of the diode D2 was about 2.3 at $R e_{\mathrm{d}}=3000$, this diodicity is even higher than the diodicity of the diode from [20] having the same planar geometry. The strong deviation can be caused by a non-similarity in an aspect ratio of both channels. The aspect ratio of the present diode channel is 0.5 , whereas for the other diode is 2.5 .

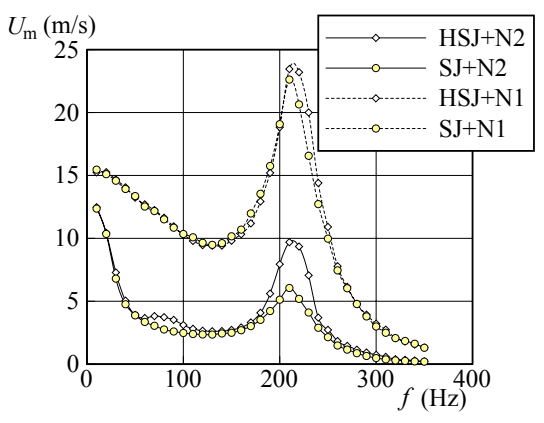

Figure 4: Velocity resonance curves for SJs and HSJs.

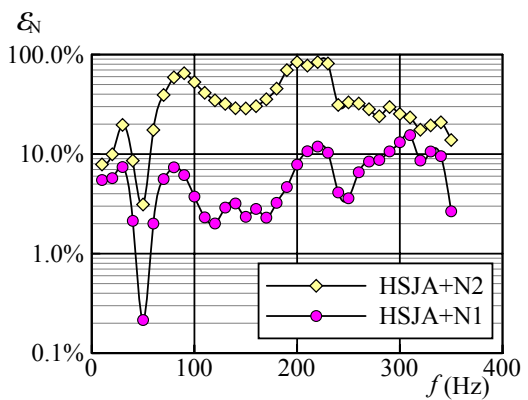

Figure 5: The measured efficiencies of both HSJA variants. 


\subsection{Velocity resonance curves}

Figure 4 compares the velocity resonance curves acquired for the HSJA and SJA measured at constant effective value of electrical current $I_{\text {eff }}=0.8 \mathrm{~A}$ (for other power details see Table 1). The velocity resonance curves are evaluated here as dependencies of the centerline time-mean velocity $U_{\mathrm{m}}=\frac{1}{T_{\mathrm{E}}} \int_{0}^{T_{\mathrm{E}}} U(t) \mathrm{d} t$ on the driving current frequency. The curves were measured with the nozzle N1 and $\mathrm{N} 2$; for nozzle N1, the experimental results for HSJ and SJ show nearly the same velocity amplitudes. The curves, however, slightly diverge behind the second velocity resonance $(210 \mathrm{~Hz})$, where the HSJ has higher velocity amplitudes, and beyond that merge again (at $260 \mathrm{~Hz}$ ).

A much larger difference between the HSJ and SJ is noticeable for the experiment with the nozzle N2. The velocity amplitude differences can be found in the range of 50 to $120 \mathrm{~Hz}$ and especially in the vicinity of the second velocity resonance $(170-270 \mathrm{~Hz})$. The largest difference is at $220 \mathrm{~Hz}$, where the velocity amplitude of HSJ is nearly 1.8 times higher than the SJ amplitude.

\subsection{Volumetric efficiency $\varepsilon_{N}$}

In Fig. 5 we can see the comparisons of the volumetric efficiencies $\varepsilon_{N}$ for HSJA with nozzle N1, or nozzle N2. The curves in Fig. 5 reach local maxima around 80-90 Hz. Other local maxima of $\varepsilon_{\mathrm{N}}$ can be found for both variants of HSJA close the second velocity resonance (around $220 \mathrm{~Hz}$ ); for the HSJA with nozzle N2, the volumetric efficiency $\varepsilon_{\mathrm{N}}$ exceeds even the value of $80 \%$ at this point.

\subsection{Velocity waveforms}

The phase-averaged velocity waveforms of SJA and HSJA are depicted in Figs. 6, 7 , and 8 at frequencies where the local maxima of volumetric efficiencies $\varepsilon_{\mathrm{N}}$ were found.

The effect of enlargement of the extrusion duration time described in [4], combined with positive velocity amplitude enhancement, is noticeable in Fig. 6, where the waveforms of the SJ and HSJ with the nozzle N1 are compared. However, much higher volumetric efficiencies were achieved for the configuration using nozzle N2; see Figs. 7, 8, where enlargement of the extrusion stroke duration time, the enhancement of the positive velocity amplitude, and the decrease of the negative velocity amplitude are apparent.

\section{Conclusion}

Two fluidic diodes were studied in this paper. The planar geometry of the first one was taken from the literature [20] and the second diode was newly developed. Functionalities of the fluidic diodes were verified by a static experiment, and their diodicities were determined. The diodicity of the first diode showed to be 


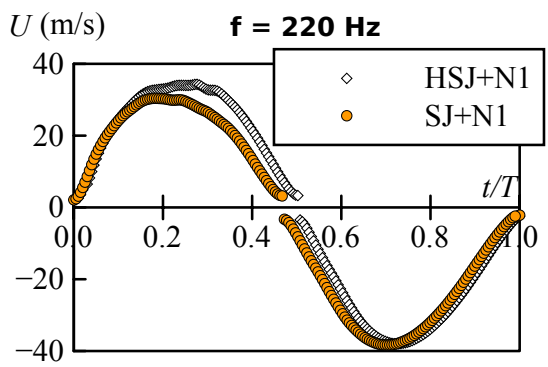

Figure 6: The velocity waveform for HSJA with short nozzle N1.

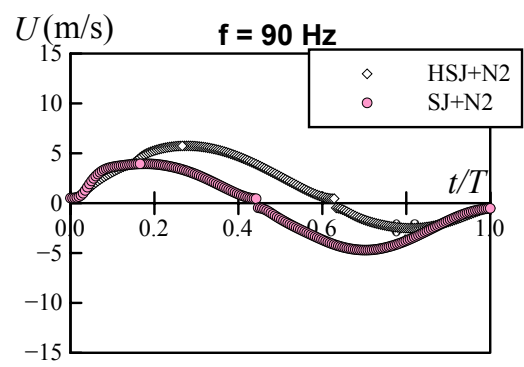

Figure 7: The velocity waveform for HSJA with long nozzle N2 acquired at $90 \mathrm{~Hz}$.

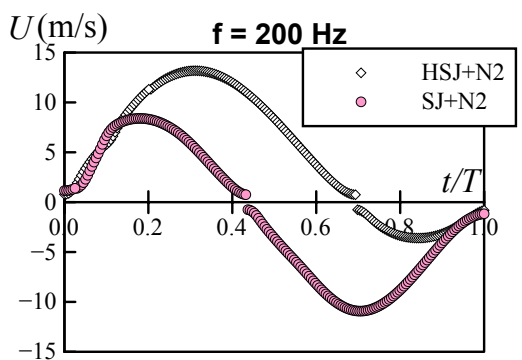

Figure 8: The velocity waveform for HSJA with long nozzle N2 acquired at $200 \mathrm{~Hz}$.

approximately 2.3 and the diodicity of the novel diode was about 3.0 (both at the Reynolds number 6000).

The novel diode was used in the HSJA, which was tested in two variants with a short nozzle and with a long nozzle. To quantify the quality of the HSJA the volumetric efficiency $\varepsilon_{\mathrm{N}}$ (defined in Eq. (2)) was measured. The volumetric efficiency was tested for both variants of the HSJA in the frequency range from 10 to $300 \mathrm{~Hz}$. A very high volumetric efficiency $\varepsilon_{\mathrm{N}}$ was achieved for the case with a longer nozzle at the second velocity resonance; the value of the efficiency exceeded $80 \%$.

\section{Acknowledgement}

We gratefully acknowledge the financial support of the GAČR P101/12/P556 and GA AV ČR (IAA200760801). 


\section{References}

[1] Trávníček, Z., Fedorchenko, A. \& Wang, A.B., An enhancement of synthetic jets by means of an integrated valveless pump. Proceedings of the Tenth Asian Congress of Fluid Mechanics (ACFMX), ed. J.J. Wijetunge, Peradeniya, pp. 535-540, 2004.

[2] Trávníček, Z., Fedorchenko, A. \& Wang, A.B., Enhancement of synthetic jets by means of an integrated valve-less pump Part I. Design of the actuator. Sensors and Actuators A: Physical, 120(1), pp. 232-240, 2005.

[3] Trávníček, Z. \& Vít, T., Hybrid synthetic jet intended for enhanced jet impingement heat/mass transfer. 13th International Heat Transfer Conference IHTC-13, Sydney, p. 12, 2006.

[4] Trávníček, Z., Vít, T. \& Tesař, V., Hybrid synthetic jets as the nonzero-netmass-flux synthetic jets. Physics of Fluids, 18(8), pp. 081701-1-081701-4, 2006.

[5] Stemme, E. \& Stemme, G., A valveless diffuser/nozzle-based fluid pump. Sensors and Actuators A: Physical, 39(2), pp. 159-167, 1993.

[6] Gerlach, T. \& Wurmus, H., Working principle and performance of the dynamic micropump. Sensors and Actuators A: Physical, 50(1-2), pp. 135140, 1995.

[7] Olsson, A., Stemme, G. \& Stemme, E., Diffuser-element design investigation for valve-less pumps. Sensors and Actuators A: Physical, 57(2), pp. 137-143, 1996.

[8] Gerlach, T., Microdiffusers as dynamic passive valves for micropump applications. Sensors and Actuators A: Physical, 69(2), pp. 181-191, 1998.

[9] Olsson, A., Larsson, O., Holn, J., Lundbladh, L., Ohman, O. \& Stemme, G., Valve-less diffuser micropumps fabricated using thermoplastic replication. Sensors and Actuators A: Physical, 64(1), pp. 63-68, 1998.

[10] Morris, C.J. \& Forster, F.K., Optimization of a circular piezoelectric bimorph for a micropump driver. Journal of Micromechanic and Microengineering, 10(3), pp. 459-465, 2000.

[11] Tesař, V., Hung, C. \& Zimmerman, W., No-moving-part hybrid-synthetic jet actuator. Sensors and Actuators A: Physical, 125(2), pp. 159-169, 2006.

[12] Tesař, V., Trávníček, Z., Kordík, J. \& Randa, Z., Experimental investigation of a fluidic actuator generating hybrid-synthetic jets. Sensors and Actuators A: Physical, 138(1), pp. 213-220, 2007.

[13] Tesař, V., Configurations of fluidic actuators for generating hybrid-synthetic jets. Sensors and Actuators A: Physical, 138(2), pp. 394-403, 2007.

[14] Trávníček, Z., Tesař, V. \& Kordík, J., Performance of synthetic jet actuators based on hybrid and double-acting principles. Journal of Visualization, 11(3), pp. 221-229, 2008.

[15] Hsu, S.S., Kordík, J., Trávníček, Z. \& Wang, A.B., The performance of hexagonally arranged hybrid synthetic jets. The 7th Pacific Symposium on Flow Visualization and Image Processing, Kaohsiung, p. 6, 2009. 
[16] Trávníček, Z., Tesař, V. \& Kordík, J., Double-acting hybrid synthetic jets with trigonally and hexagonally arranged nozzles. 9th International Conference on Fluid Control, Measurements, and Visualization (FLUCOME9), Tallahassee, p. 10, 2007.

[17] Yastrebova, E.V., Fluidic diodes (review). Automatika i Telemechanika, 3, pp. 101-106, 1971.

[18] Bradel, R.L., The diodicity mechanism of Tesla-type no-moving-parts valves. $\mathrm{PhD}$. thesis, University of Washington, Washington, 2000.

[19] Forster, F.K. \& Williams, B.E., Parametric design of fixed-geometry microvalves-the tesser valve. ASME International Mechanical Engineering Congress \& Exposition, IMECE2002, IMECE2002-33628: New Orleans, p. 7, 2002.

[20] Gamboa, A.R., Morris, C.J. \& Forster, F.K., Improvements in fixed-valve micropump performance through shape optimization of valves. Transactions of the ASME - Journal of Fluids Engineering, 127(2), pp. 339-346, 2005.

[21] Kordík, J., Theoretical and experimental analysis of synthetic and hybrid synthetic jet actuators. PhD. thesis, Czech Technical University, Prague, 2011. 